\title{
Cayley Cages
}

\section{Geoffrey Exoo • Robert Jajcay • Jozef Širáň}

Received: 1 September 2011 / Accepted: 17 September 2012 / Published online: 4 October 2012

(C) Springer Science+Business Media New York 2012

\begin{abstract}
A $(k, g)$-Cayley cage is a $k$-regular Cayley graph of girth $g$ and smallest possible order. We present an explicit construction of $(k, g)$-Cayley graphs for all parameters $k \geq 2$ and $g \geq 3$ and generalize this construction to show that many wellknown small $k$-regular graphs of girth $g$ can be constructed in this way. We also establish connections between this construction and topological graph theory, and address the question of the order of $(k, g)$-Cayley cages.
\end{abstract}

Keywords Cage $\cdot$ Cayley graph $\cdot$ Girth

\section{Introduction}

Throughout our paper, a $k$-regular graph of girth $g$ is called a $(k, g)$-graph, a smallest $(k, g)$-graph is called a cage, and the order of a $(k, g)$-cage is denoted by $n(k, g)$. The existence of $(k, g)$-graphs for all pairs $k \geq 2$ and $g \geq 3$ has been established in the 1960s twice in almost simultaneous papers by Erdős and Sachs [4] and by Sachs alone [12]. While the proof in [4] is essentially nonconstructive, the proof in [12] relies on a construction we call in this paper a generalized truncation. To this day, Sachs' construction is one of the few universal constructions (i.e., constructions that work

G. Exoo $\cdot$ R. Jajcay $(\bowtie)$

Department of Mathematics and Computer Science, Indiana State University, Terre Haute, IN 47809,

USA

e-mail: robert.jajcay@indstate.edu

G. Exoo

e-mail: ge@cs.indstate.edu

J. Širáň

Department of Mathematics, SvF, Slovak University of Technology, Radlinského 11, 81368

Bratislava, Slovakia

e-mail: siran@math.sk 
for any choice of $k$ and $g$ ) completely void of algebraic arguments. This is in stark contrast with the fact that the majority of the best $(k, g)$-constructions (constructions resulting in graphs of the smallest order) both rely on the use of algebraic arguments and result in graphs possessing a high level of symmetry.

In view of the importance of the role of symmetry in constructions of small $(k, g)$ graphs, we set to investigate the girth/order relation for the subclass of the class of vertex-transitive graphs called Cayley graphs. Cayley graphs are vertex-transitive graphs that admit a group of automorphisms $\Gamma$ that acts regularly on the vertices of the graph (i.e., the action of $\Gamma$ on the set of the vertices of the graph is transitive, and the stabilizer of any vertex in $\Gamma$ is trivial). Equivalently, if $\Gamma$ is a group generated by a set of elements $X$ that does not contain the identity of $\Gamma$ and contains the inverse of $x$ for each $x \in X$, then the Cayley graph $\operatorname{Cay}(\Gamma, X)$ is the graph with vertex set $\Gamma$ and the edge set $\{\{g, g x\} \mid g \in \Gamma, x \in X\}$.

The existence of Cayley graphs of any given degree $k \geq 2$ and girth $g \geq 3$ has been recently established in [8]. The proof takes advantage of an infinite family of (infinite) Cayley maps constructed by Šiagiová and Watkins [13] and the fact that the automorphism groups of these maps are residually finite. It is important to note, however, that the construction presented in [8] relies on the existence of certain finiteindex normal subgroups of residually finite groups, which are known to exist, but very little is known about finding them or about the value of their indices. Thus, the construction in [8] is not explicit. On the other hand, an explicit construction of $k$ regular Cayley graphs of girth at least $g$ due to Biggs [2] has been known since the 1990 s, but repeated attempts at adjusting this construction to produce graphs of girth exactly $g$ have been unsuccessful.

We remedy this situation in the first part of our paper, where we present an explicit construction of $(k, g)$-Cayley graphs for any pair of parameters $k$ and $g$. These will be defined via their action on special $(k, g)$-graphs constructed using the original truncation method of Sachs.

The next section contains a generalization of this construction to more general settings where the resulting graphs do not have to be vertex-transitive-we show that a number of well-known graphs can be constructed this way. We also investigate further connections of our techniques to topological graph theory, namely to the theory of permutation and voltage lifts.

In the last two parts of our paper, we address the issue of the order of the constructed graphs, discuss the possibilities for constructions of smaller graphs, and finally focus on Cayley cages, smallest $k$-regular Cayley graphs of girth $g$.

\section{Construction of Cayley graphs of given degree and girth}

Map truncation is a procedure often used in topological graph theory whereby the vertices of a map are replaced by cycles. As this method allows for a great deal of control over the cycles of the truncated map, it has also been repeatedly used in the construction of graphs of given degree and girth. Most notably, it has been employed in a 1963 paper of Sachs [12] to prove the existence of a finite $k$-regular graph of girth $g$ for every pair $k \geq 2, g \geq 3$. Since Sachs' construction does not generally result in 
vertex-transitive graphs, we find it a bit surprising that we were able to employ Sachs' construction to construct $k$-regular Cayley graphs of prescribed girth.

In what follows, we rely on a restatement of Sachs' construction presented in [5]. Let $G$ be a finite $k$-regular graph. To initialize our construction, we associate each edge of $G$ with a pair of opposing darts, both connecting the two end-points of the edge but each starting at a different end, and let $D(G)$ denote the set of darts obtained in this way; thus, $|D(G)|=2|E(G)|$. A vertex-neighborhood labeling of $G$ is any function $\rho$ mapping $D(G)$ onto the set $\{1,2, \ldots, k\}$ that satisfies the property that for any vertex $v$ of $G$, the darts emanating from $v$ all receive different labels ( $\rho$ maps the darts emanating from each vertex onto the set $\{1,2,3, \ldots, k\})$.

Let $H$ be a graph of order $k$ with vertex set $V(H)=\left\{u_{1}, u_{2}, \ldots, u_{k}\right\}$. The generalized truncation of a $k$-regular graph $G$ with a vertex-neighborhood labeling $\rho$ by the graph $H$ is the graph $T(G, \rho, H)$ obtained from $G$ by replacing the vertices of $G$ by copies of $H$ according to the rule that the vertex $u_{i}$ is attached to the dart labeled $i$ for $1 \leq i \leq k$. More formally, the vertex set of the truncated graph $T(G, \rho, H)$ consists of the vertices $u_{i}^{v}$, where $i$ runs from 1 through $k$, and $v$ runs through the set of vertices $v \in V(G)$ (and hence the order of the truncated graphs is the product of the orders of the graphs $G$ and $H$ ). Any two vertices $u_{i}^{v}, u_{j}^{v}$ are joined by an edge if and only if $u_{i}$ and $u_{j}$ were adjacent in $H$, and any two vertices $u_{i}^{v}, u_{j}^{w}, v \neq w$, are joined by an edge if and only if $v$ and $w$ were adjacent in $G$ and the corresponding labels of the darts $(v, w)$ and $(w, v)$ are $\rho(v, w)=j$ and $\rho(w, v)=i$.

The following easy lemma is stated for future reference.

Lemma 1 Let $T(G, \rho, H)$ be a generalized truncation of a $k$-regular graph $G$ by a graph $H$ of order $k$. The subgraphs of $T(G, \rho, H)$ induced by the sets $\left\{u_{1}^{v}, u_{2}^{v}, \ldots, u_{k}^{v}\right\}, v \in V(G)$, are nonoverlapping isomorphic copies of $H$ that partition the vertex set of the truncation, and the edges that connect different copies of $H$ form a 1-factor of the truncation.

The relevance of the generalized truncation for the girth of the resulting graphs is summarized in the following theorem.

Theorem 1 ([3]) Let $G$ be a finite $(k, g)$-graph with a vertex-neighborhood labeling $\rho$, and let $H$ be a $\left(k^{\prime}, g^{\prime}\right)$-graph of order $k$. The generalized truncation graph $T(G, \rho, H)$ is a $\left(k^{\prime}+1\right)$-regular graph of girth not smaller than $\min \left\{2 g, g^{\prime}\right\}$, and if $g^{\prime} \leq 2 g$, then $g^{\prime}$ is the exact girth of $T(G, \rho, H)$.

With the help of the above result, we are ready to state our main theorem. A product of group elements $x_{i_{1}} x_{i_{2}} \ldots x_{i_{s}}$ is called nonreversing if it contains no pair of consecutive mutually inverse elements: $x_{i_{j}} \neq\left(x_{i_{j+1}}\right)^{-1}, 1 \leq j \leq s-1$. Nonreversing products of elements from $X$ in a Cayley graph $\operatorname{Cay}(\Gamma, X)$ represent walks without edges traveled back and forth in two consecutive moves.

Theorem 2 For every pair $k \geq 2$ and $g \geq 3$, there exists a Cayley graph of degree $k$ and girth $g$. 
Proof We proceed by induction on the degree $k$.

First, note that the $g$-cycle $\mathcal{C}_{g}$ is isomorphic to the Cayley graph $\operatorname{Cay}\left(\mathbb{Z}_{g}\right.$, $\{1, g-1\})$. Thus, there exists a 2-regular Cayley graph of girth $g$ for every $g \geq 3$.

Next, assume that $k=3$ and $g \geq 3$ and let $F$ be a $g$-regular graph of girth $g^{\prime}>2 g$ (the existence of which is guaranteed, for example, by the results of Sachs [12]). Let $\rho$ be any vertex-neighborhood labeling of $F$, and let $G$ be the generalized truncation $T\left(F, \rho, \mathcal{C}_{g}\right.$ ) (where $\mathcal{C}_{g}$ is a cycle of length $g$ ). Then, according to Lemma 1 , these $g$-cycles of $G$ form a partition of $G$, i.e., every vertex of $G$ belongs to exactly one $g$-cycle of $G$. Next, choose for each of the $g$-cycles of $G$ one of the two possible orientations and define a permutation $\sigma$ on $V(G)$ to be the disjoint union of the $g$ cycles of $G$ ordered according to the chosen orientations. Then $\sigma$ (and hence $\sigma^{-1}$ ) is a semiregular permutation of $V(G)$ with order $g \geq 3$. Using Lemma 1 once more, note that the edges of $G$ not included in the $g$-cycles of $G$ form a 1-factor and take $\delta$ to be the involutory permutation of $V(G)$ swapping the end-vertices of the edges of this 1 -factor.

Let $\Gamma=\langle\sigma, \delta\rangle$ be the finite permutation group generated by the permutations $\sigma$ and $\delta$ acting on $V(G)$. We claim that the 3-regular Cayley graph $H=$ $\operatorname{Cay}\left(\Gamma,\left\{\sigma, \sigma^{-1}, \delta\right\}\right)$ has girth $g$. As observed above, $\sigma$ is an element of order $g$, and hence the Cayley graph $H$ contains cycles of length $g$. It remains to prove that $H$ does not contain cycles of length smaller than $g$. To see this, note that every cycle of $H$ other than the above cycles labeled exclusively by $\sigma$ (which are known to be of length $g$ ) is associated with a word

$$
\sigma^{e_{1}} \delta \sigma^{e_{2}} \delta \cdots \sigma^{e_{r}} \delta=1_{\Gamma}
$$

of length $\ell=\left|e_{1}\right|+\left|e_{2}\right|+\cdots+\left|e_{r}\right|+r$. Then

$$
\sigma^{e_{1}} \delta \sigma^{e_{2}} \delta \cdots \sigma^{e_{r}} \delta(v)=v
$$

for all $v \in V(G)$. It is easy to see from the definition of the permutations $\sigma$ and $\delta$ that every vertex $v \in V(G)$ is adjacent to both $\sigma(v)$ and $\delta(v)$, and neither one of these vertices is equal to $v$. Thus, the sequence

$$
v, \delta(v), \sigma \delta(v), \ldots, \sigma^{e_{r}} \delta(v), \ldots, \sigma^{e_{1}} \delta \sigma^{e_{2}} \delta \cdots \sigma^{e_{r}} \delta(v)
$$

is a nonreversing sequence of vertices of the original graph $G$ that starts and ends with the same vertex $v$, and any two consecutive vertices in the sequence are adjacent. It follows that this sequence must contain a cycle of $G$ which has girth $g$. Hence, the length $\ell$ of this sequence is at least $g$, and every cycle in $H$ must be of length at least $g$. That completes the proof of the claim that $H$ is a 3-regular Cayley graph of girth $g$.

For the induction step, assume that $k>3$ and $g \geq 3$ and that there exists a Cayley graph $H=\operatorname{Cay}(\Lambda, X)$ of degree $k-1$, girth $g$, and order $n$. Let $F$ be any graph of degree $n$ and girth larger than $2 g$, and let $G$ be the truncation of $F$ by $H$ (with an arbitrary vertex-neighborhood labeling). Then $G$ is a $k$-regular graph of girth $g$ by Lemma 1 , and Lemma 1 implies that each edge of $G$ either belongs to a copy of $H$ and thus is labeled by a generator $x \in X$ or belongs to a 1 -factor of $G$. For every 
$x \in X$, define $\sigma_{x}: \lambda^{v} \rightarrow(\lambda x)^{v}$ for all $\lambda \in \Lambda$ and $v \in V(F)$ to be the permutation of $V(G)$ acting locally on the copies of $H$ via left multiplication by $x$. Let $\delta$ be the involutory permutation swapping the end-points of the edges included in the 1-factor joining the copies of $H$. If we now take $\Gamma$ to be the group generated by the elements $\sigma_{x}, x \in X$, and the involution $\delta$, an argument identical to the one used above shows that $\operatorname{Cay}\left(\Gamma,\left\{\sigma_{x} \mid x \in X\right\} \cup\{\delta\}\right)$ is a Cayley graph of degree $k$ and girth $g$.

\section{Groups from graphs and graphs from groups}

Many constructions of groups from graphs and graphs from groups are known (Cayley graphs are certainly one such example). In this section, we generalize the construction of a group from a regular graph presented in the proof of Theorem 2 and show that under certain circumstance it may be reversed and leads to constructions of small regular graphs of given girths.

Although it is often assumed to be a part of the definition, we underline here the fact that the 1-factors (or perfect matchings) and the 2-factors considered in our paper cover all vertices of their respective graphs.

To construct groups from graphs, we introduce the following notation. Let $G$ be a graph, and let $F \subseteq E(G)$ be a 1 -factor of $G$. The involution $\delta_{F}$ associated with the 1-factor $F$ is the permutation of $V(G)$ swapping the end points of the edges in $F$. In the case where $F$ is a 2 -factor of $G$ (that partitions $V(G)$ into nonoverlapping cycles), choose an orientation for each cycle in $F$ and let the permutation $\sigma_{F}$ associated with the (oriented) 2-factor $F$ be the permutation of $V(G)$ mapping each vertex $v \in V(G)$ to its neighbor in $F$ (with respect to the chosen orientation).

The proof of the following theorem follows along the same lines as the proof of Theorem 2.

Theorem 3 Let $G$ be a $k$-regular graph of girth $g$ whose edge set can be partitioned into a family $\mathcal{F}$ of $k_{1} 1$-factors, $F_{i}, 1 \leq i \leq k_{1}$, and $k_{2}$ oriented 2 -factors $F_{i}, k_{1}+1 \leq$ $i \leq k_{1}+k_{2}$ (where $k_{1}+2 k_{2}=k$ ). If $\Gamma_{\mathcal{F}}$ is the finite permutation group acting on the set $V(G)$ generated by the set

$X=\left\{\delta_{F_{i}} \mid 1 \leq i \leq k_{1}\right\} \cup\left\{\sigma_{F_{i}} \mid k_{1}+1 \leq i \leq k_{1}+k_{2}\right\} \cup\left\{\sigma_{F_{i}}^{-1} \mid k_{1}+1 \leq i \leq k_{1}+k_{2}\right\}$,

then the Cayley graph $\operatorname{Cay}\left(\Gamma_{\mathcal{F}}, X\right)$ is $k$-regular of girth at least $g$.

Unlike the case considered in Theorem 2, the girth of the resulting Cayley graph can be bigger than the original girth $g$. Since all the cycles included in the 2-factors of $G$ used in the proof of Theorem 2 were of equal length $g$, the orders of the permutations induced by the 2-factors of $G$ were also $g$. In the general situation, where our 2 -factors are allowed to contain cycles of different lengths, the corresponding orders of the permutations they give rise to are equal to the least common multiple of the lengths of the cycles included. We summarize these observations as follows.

Corollary 1 Let $G, \mathcal{F}$, and $\Gamma_{\mathcal{F}}$ be the same as those in Theorem 3. For each $k_{1}+1 \leq$ $i \leq k_{1}+k_{2}$, let $o_{i}$ denote the least common multiple of the lengths of cycles included 
in the 2-factor $F_{i}$. Then the girth of the Cayley graph $C a y\left(\Gamma_{\mathcal{F}}, X\right)$ is at least $g$ and at most $\min \left\{o_{i} \mid k_{1}+1 \leq i \leq k_{1}+k_{2}\right\}$.

Consequently, if all the 2 -factors in $\mathcal{F}$ consist of cycles of length $g$, the resulting Cayley graph $\operatorname{Cay}\left(\Gamma_{\mathcal{F}}, X\right)$ is of girth $g$.

Any finite Cayley graph $\operatorname{Cay}(\Gamma, Y)$ allows for a partition into 1- and 2-cycles like those in Theorem 3. To see this, observe that every edge of a Cayley graph is associated with either an involution or a pair of mutually inverse elements in $Y$. Moreover, the edges labeled by an involution form a 1-factor, and the edges labeled by a pair of inverses form a 2-factor of the Cayley graph. Thus, the labeling determined by the elements of $Y$ determines a partition of the edge set of $\operatorname{Cay}(\Gamma, Y)$ into 1- and 2 -factors. We call this partition a Cayley partition of $\operatorname{Cay}(\Gamma, Y)$. If $\mathcal{F}$ is a Cayley partition of $\operatorname{Cay}(\Gamma, Y)$, we can choose one element from every pair of inverses in $Y$ and orient the cycles of the corresponding 2-factor in agreement with the left multiplication action of this element. The resulting group $\Gamma_{\mathcal{F}}$ is isomorphic to the group $\Gamma, \Gamma_{\mathcal{F}} \cong \Gamma$, and the Cayley graph $\operatorname{Cay}\left(\Gamma_{\mathcal{F}}, X\right)$ coincides with the original graph $\operatorname{Cay}(\Gamma, Y), \operatorname{Cay}\left(\Gamma_{\mathcal{F}}, X\right) \cong \operatorname{Cay}(\Gamma, Y)$. The elements of $X$ (as permutations of the vertex set of $\operatorname{Cay}(\Gamma, Y))$ are graph automorphisms of $\operatorname{Cay}(\Gamma, Y)$ induced by left multiplication by elements of $Y$. If, on the other hand, we do not start from a Cayley graph or a Cayley partition, the permutations in $\Gamma_{\mathcal{F}}$ do not necessarily turn out to be graph automorphisms of the starting graph. In fact, aside from the Cayley partitions, we do not know of other cases where the group $\Gamma_{\mathcal{F}}$ is a subgroup of the group $\operatorname{Aut}(G)$.

Also, if we choose the directions of the cycles in a way that does not agree with the direction induced by the left multiplication by the elements of $Y$, the resulting graphs may not be isomorphic. Consider, for example, the 8-vertex prism $\operatorname{Cay}(\Gamma, Y)$ with

$$
Y=\{(1,5)(2,6)(3,7)(4,8),(1,2,3,4)(5,6,7,8),(1,4,3,2)(5,8,7,6)\},
$$

in which the group $\Gamma=\langle Y\rangle \cong \mathbb{Z}_{2} \times \mathbb{Z}_{4}$ is abelian. However, the group generated by the elements $(1,5)(2,6)(3,7)(4,8),(1,2,3,4)(5,8,7,6)$, and $(1,4,3,2)(5,6,7,8)$ (where the second cycle is reversed) is the non-abelian dihedral group $\mathbb{D}_{4}$.

To consider a more interesting example, take $\mathcal{P}$ to be the Petersen graph, and let $F_{1}$ be the 1-factor $\{1,6\},\{2,7\},\{3,8\},\{4,9\},\{5,10\}$, and $F_{2}$ be the oriented 2factor $\{1,2,3,4,5\},\{6,8,10,7,9\}$. The resulting group $\Gamma_{\mathcal{F}}$ generated by the set $X$ consisting of the permutations

$$
(1,6)(2,7)(3,8)(4,9)(5,10),(1,2,3,4,5)(6,8,10,7,9),(1,5,4,3,2)(6,9,7,10,8)
$$

is of order 50 and is isomorphic to a direct product of $\mathbb{Z}_{5}$ and the dihedral group of 10 elements $\mathbb{D}_{5}$. Interestingly, the 3-regular graph $\operatorname{Cay}\left(\Gamma_{\mathcal{F}}, X\right)$ of girth 5 (see Fig. 1) is also the smallest Cayley graph of degree 3 and girth 5. This way the smallest 3 regular graph of girth 5 gives rise to the smallest 3-regular Cayley graph of girth 5 . As can be seen from the table of small $(k, g)$-Cayley cages included at the end of our paper, this smallest 3-regular Cayley graph of girth 5 is larger than the smallest 3-regular Cayley graphs for girths 6, 7, and 8. 
Fig. 1 Smallest 3-regular Cayley graph of girth 5

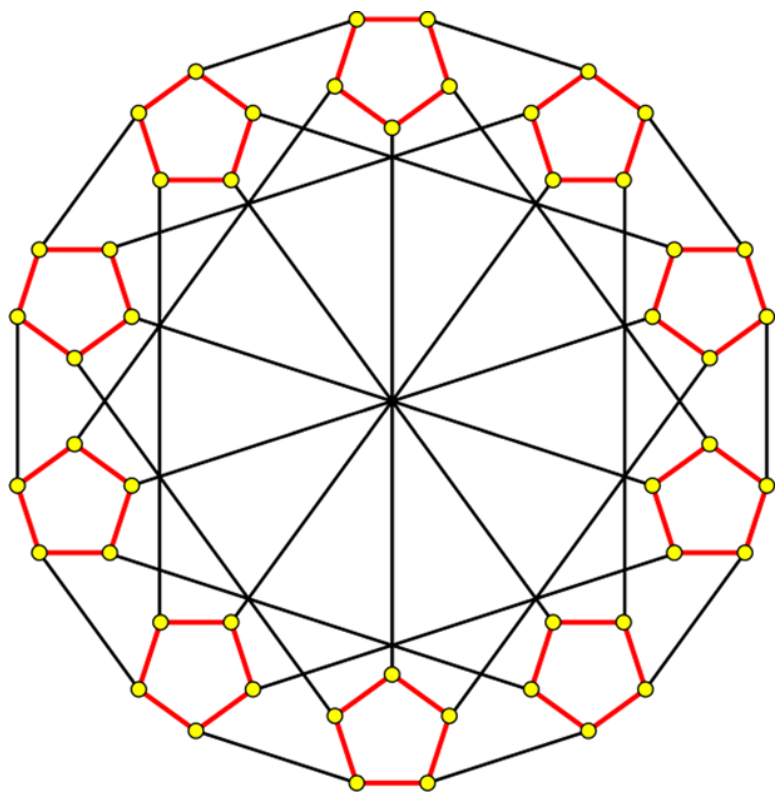

Since the order of the full automorphism group of the Petersen graph, $\operatorname{Aut}(\mathcal{P})$, is equal to $120, \Gamma_{\mathcal{F}}$ is not a group of automorphisms of $\mathcal{P}$. Despite this fact, one can reverse the above construction and construct the Petersen graph from the larger graph $\operatorname{Cay}\left(\Gamma_{\mathcal{F}}, X\right)$. We generalize this reversed construction as follows.

Theorem 4 Let $C a y(\Gamma, X)$ be a k-regular graph of girth $g$. Suppose that $\Gamma$ has a permutation representation $\gamma \rightarrow \sigma_{\gamma}, \gamma \in \Gamma$, on a set $V$, satisfying the property that no nonreversing product of the permutations $\sigma_{x}, x \in X$, of length smaller that $g$ fixes a vertex $v \in V$, and for every $v \in V$, the images $\sigma_{x}(v)$ are all different. Then the graph $G_{\Gamma}$ with vertex set $V$ and edge set $E=\left\{\left\{v, \sigma_{x}(v)\right\} \mid v \in V, x \in X\right\}$ is $k$-regular of girth $g$.

Proof The degree of $G_{\Gamma}$ is equal to $k$, the order of the set $X$. Since the girth of $\operatorname{Cay}(\Gamma, X)$ is assumed to be $g$, there must exist a nonreversing word $x_{i_{1}} x_{i_{2}} \ldots x_{i_{g}}$, $x_{i_{j}} \in X$ for all $1 \leq j \leq g$ that is equal to $1_{\Gamma}$. It follows that $\sigma_{x_{i_{1}}} \sigma_{x_{i_{2}}} \ldots \sigma_{x_{i_{g}}}(v)=v$ (for any $v \in V$ ), and so the path determined in $G_{\Gamma}$ by the permutations $\sigma_{x_{i}}$ must contain a cycle of $G_{\Gamma}$, and hence the girth of $G_{\Gamma}$ is not larger than $g$. If $G_{\Gamma}$ contained a cycle of length smaller than $g$, its labels would spell a nonreversing word composed of the elements $\sigma_{x_{i j}}$ that would fix any vertex of the cycle, which contradicts the assumption of the theorem.

Notice that we do not require the action of $\Gamma$ on $V$ to be transitive. Observe also that the resulting graph $G_{\Gamma}$ is connected if and only if the action of $\Gamma$ is transitive on $V$ (and otherwise $G_{\Gamma}$ consists of components that coincide with the orbits of $\Gamma$ on $V)$. 
Even though the examples we have used so far may seem to suggest the opposite, the transitivity of the action of $\Gamma$ on $V$ does not imply the vertex-transitivity of the resulting graphs. To see this, consider the family of generalized Petersen graphs $\mathcal{P}(n, r), 3 \leq n, 1 \leq r \leq n-1$, with vertex sets

$$
V(\mathcal{P}(n, r))=\{(i, j) \mid 1 \leq i \leq n, 0 \leq j \leq 1\}
$$

and edge sets

$$
\begin{aligned}
E(\mathcal{P}(n, r))= & \{\{(i, 0),(i+1,0)\} \mid 1 \leq i \leq n\} \\
& \cup\{\{(i, 1),(i+r, 1)\} \mid 1 \leq i \leq n\} \\
& \cup\{\{(i, 0),(i, 1)\} \mid 1 \leq i \leq n\}
\end{aligned}
$$

(with the additions performed modulo $n$ ). All generalized Petersen graphs are connected and allow for an edge partition into a 1 -factor and a 2 -factor, with the 1 -factor consisting of the edges $\{\{(i, 0),(i, 1)\} \mid 1 \leq i \leq n\}$, and the 2-factor consisting of the remaining edges. Thus, for each pair of parameters $(n, r)$, this partition determines a Cayley graph $\operatorname{Cay}\left(\Gamma_{\mathcal{P}(n, r)}, X\right)$. As all generalized Petersen graphs are connected, the actions of the corresponding groups $\Gamma_{\mathcal{P}(n, r)}$ are transitive, and thus the generalized Petersen graphs can all be "reverse constructed" from the groups $\Gamma_{\mathcal{P}(n, r)}$ along the lines described in Theorem 4. Even though all the actions of the groups $\Gamma_{\mathcal{P}(n, r)}$ are vertex-transitive, because they do not respect the edges of the resulting generalized Petersen graphs, the resulting graphs are not necessarily vertex-transitive. In fact, it is well known that not all generalized Petersen graphs are vertex-transitive [11].

Although the assumptions of Theorem 4 seem very restrictive, the example of the Petersen graph constructed from the Cayley graph of order 50 suggests that they may be satisfied. Thus, one may be able to construct a small (not necessarily vertextransitive) $k$-regular graph of girth $g$ from a larger $(k, g)$-Cayley graph. We conclude this section with one such construction.

Theorem 5 Let $H=C a y(\Gamma, X)$ be a k-regular Cayley graph of girth $g \geq 3$. Let $3 \leq h<g$, and let $N=N_{h}\left(1_{\Gamma}\right)$ be the set of elements of $\Gamma$ whose distance from the vertex $1_{\Gamma}$ in $H$ is less than or equal to $h$. If there exists a subgroup $\Lambda$ of $\Gamma$ whose intersection with the set $N^{\Gamma}$, the union of the conjugates of $N$ in $\Gamma$, consists of $1_{\Gamma}$ alone, then there exists a k-regular graph of girth at least $h$, and at most $g$, whose vertex set consists of the left cosets of $\Lambda$ in $\Gamma$.

Proof Suppose that $\Gamma$ and $\Lambda$ are as described, and recall that $\Gamma$ acts on the left cosets of $\Lambda$ via an action induced by left multiplication: $(a \Lambda)^{g}=(g a) \Lambda$. Thus, to complete the proof of our theorem, it is enough to show that this action of $\Gamma$ on the cosets of $\Lambda$ satisfies the conditions of Theorem 4 . If $(a \Lambda)^{x_{i}}=(a \Lambda)^{x_{j}}$ for some $a \in \Gamma$ and $x_{i} \neq x_{j} \in X$, we could conclude that $a^{-1} x_{j}^{-1} x_{i} a \in \Lambda$, which is, however, only possible if $x_{i}=x_{j}$. Hence all the neighbors of a coset $a \Lambda$ are different (and, by the same argument, different from $a \Lambda$ as well). If a product $x_{i_{1}} x_{i_{2}} \ldots x_{i_{s}}$ of $s \leq h$ elements in $X$ fixed a coset $a \Lambda$, we would obtain another contradiction with the assumption that conjugates of such elements do not belong to $\Lambda$. 
Fig. 2 The McGee graph

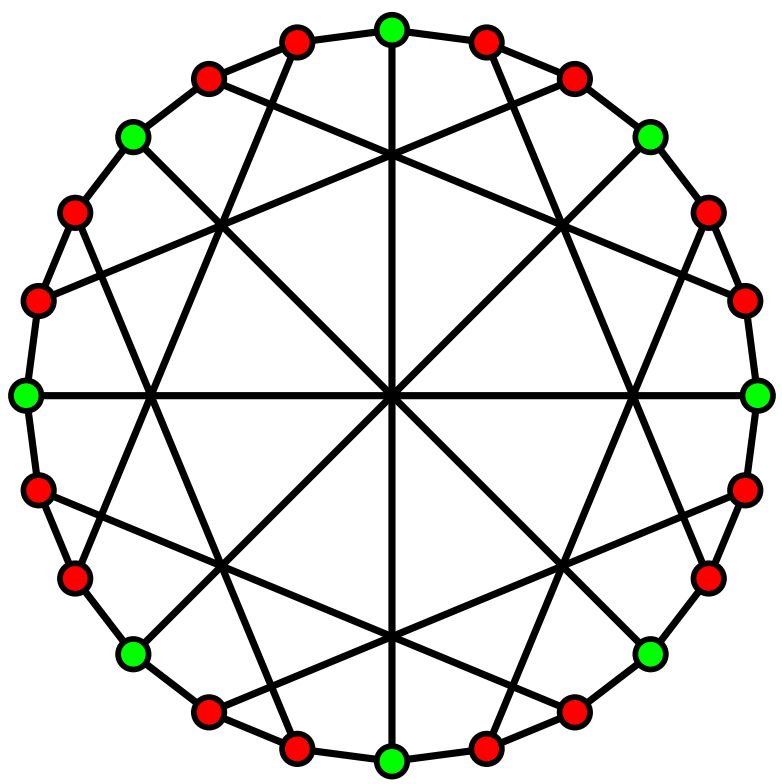

Since the vertices of the graph we construct in the above proof are the left cosets of a subgroup, the reader familiar with Schreier coset graphs may be reminded of these. Our graphs are not Schreier coset graphs as our graphs do not possess pairs of opposing darts. For more details, consult, for example, [6].

There is also a connection between the factorization used in Theorem 5 and a factorization technique often used in the theory of residually finite groups. An (infinite) group $\Gamma$ is called residually finite if for any finite subset $S$ of $\Gamma$ that does not contain the identity $1_{\Gamma}$, there exists a normal finite-index subgroup $\Delta$ of $\Gamma$ that avoids $S$ : $\Delta \cap S=\emptyset$ (see, e.g., [1]). The original proof of the existence of a finite $(k, g)$-Cayley graph for any pair of parameters $(k, g)$ in [8] took advantage of the residual finiteness of the automorphism groups of certain infinite $(k, g)$-Cayley maps and relied on factoring out finite-index subgroups that did not change the local $(k, g)$-structure of these infinite maps. Notably, the construction in Theorem 5 does not require for the group to be factored out to be normal in $\Gamma$.

We illustrate the use of Theorem 5 in the construction of the $(3,7)$-cage, the McGee graph (see Fig. 2). It is the first trivalent cage that is neither a Moore nor a vertex-transitive graph.

The McGee graph has order 24 (exceeding the Moore bound by 2, see Sect. 5), an automorphism group of order 32, and two vertex orbits of sizes 8 and 16. These orbits are indicated by the vertex colorings in the figure.

One can construct the McGee graph as a coset graph using a group $\Gamma$ of order 48 and a subgroup of order 2 . To construct $\Gamma$, one begins with the triple direct product $\mathbb{Z}_{4} \times \mathbb{Z}_{2} \times \mathbb{Z}_{2}$. Elements in this product are represented by triples $\left(x_{1}, x_{2}, x_{3}\right)$ where $0 \leq x_{1}<4,0 \leq x_{2}<2$, and $0 \leq x_{3}<2$. The group $\Gamma$ is a semidirect product $\left(\mathbb{Z}_{4} \times\right.$ $\left.\mathbb{Z}_{2} \times \mathbb{Z}_{2}\right) \rtimes_{\phi} \mathbb{Z}_{3}$. The automorphism $\phi$ is defined by $\phi\left(x_{1}, x_{2}, x_{3}\right)=\left(x_{1}, x_{3}, x_{2}+\right.$ 
Fig. 3 A Cayley Graph of the group $\left(\mathbb{Z}_{4} \times \mathbb{Z}_{2} \times \mathbb{Z}_{2}\right) \rtimes_{\phi} \mathbb{Z}_{3}$

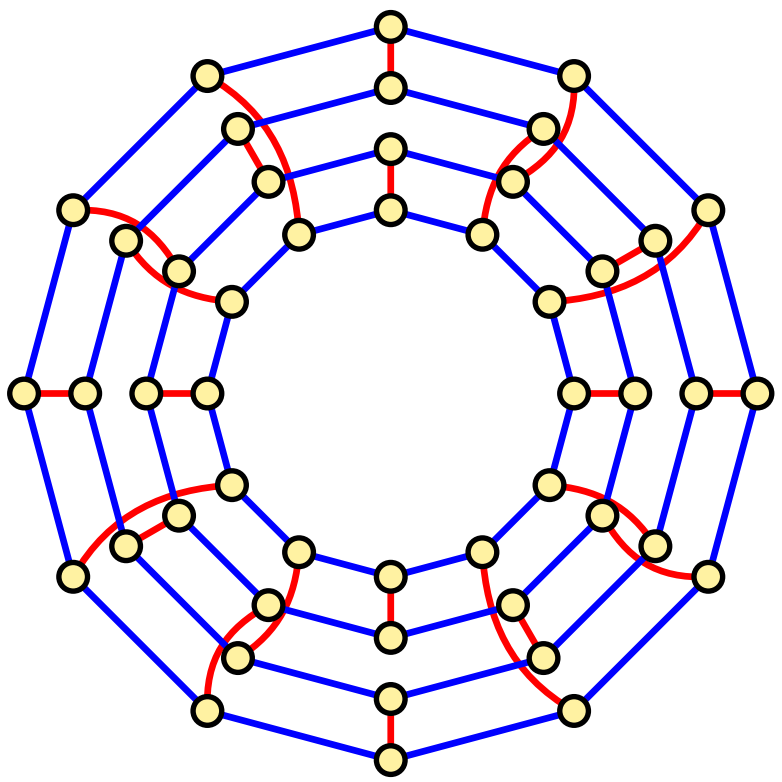

$\left.x_{3}\right)$, with $\mathbb{Z}_{2}$ addition in the third coordinate. ${ }^{1}$ The elements of $\Gamma$ are represented as quadruples $\left(x_{1}, x_{2}, x_{3}, x_{4}\right)$ where $x_{4} \in \mathbb{Z}_{3}$. The generators for $\Gamma$ used in the Cayley double cover of the McGee graph are the involution $\alpha=(0,0,1,0)$ along with $\beta=$ $(1,0,0,1)$ and its inverse $\beta^{-1}=(3,0,0,2)$. The graph is shown in Fig. 3, where $\alpha$ edges are colored in blue, and $\beta$ edges in red.

The subgroup of order 2 that will be factored out to obtain the coset graph isomorphic to the McGee graph is generated by the involution $(2,0,1,0)$. This element is at maximum distance from the identity in the Cayley graph.

We conclude this section by relating the above to the concept of a permutation voltage graph introduced by Gross and Tucker [7]. Let $G=(V, E)$ be a finite graph that may have loops, multiple edges, and semiedges. Associate each loop and edge of $G$ with two opposing darts, and each semiedge with a single dart with no end, and let $D(G)$ denote the set of darts of $G$. Let $\Gamma$ be a permutation group acting on a set $W$, and let $\Phi: D(G) \rightarrow \Gamma$ be a mapping from the set of darts of $G$ into the group $\Gamma$ satisfying the condition that the elements assigned to opposing darts are mutual inverses. Then $\Phi$ is called the permutation voltage assignment, and the permutation voltage lift $G^{\Phi}$ of $G$ by the permutation voltage assignment $\Phi$ is a graph with vertex set $V \times W$ and the adjacency relation making $\left(v_{i}, w_{i}\right)$ adjacent to $\left(v_{j}, w_{j}\right)$ if and only if there exists a dart $e$ from $v_{i}$ to $v_{j}$ in $D(G)$, and $\Phi(e)\left(w_{i}\right)=w_{j}$. The lift $G^{\Phi}$ is the cover of the graph $G$, and in the context of the theory of topological 1-complexes, the mapping from $G^{\Phi}$ onto $G$ is a covering projection and $G^{\Phi}$ is a covering space of $G$.

The following description of all graphs admitting the edge partitions required above is not hard to deduce from [6] and [14].

\footnotetext{
${ }^{1}$ In fact, any order 3 automorphism of $\mathbb{Z}_{4} \times \mathbb{Z}_{2} \times \mathbb{Z}_{2}$ will produce the same group.
} 
Theorem 6 Let $G$ be a $k$-regular graph whose edge set can be partitioned into a family $\mathcal{F}$ of $k_{1} 1$-factors $F_{i}, 1 \leq i \leq k_{1}$, and $k_{2}$ oriented 2 -factors $F_{i}, k_{1}+1 \leq i \leq k_{1}+k_{2}$, with $k_{1}+2 k_{2}=k$. Let the group $\Gamma_{\mathcal{F}}$ and its generators $\delta_{F_{i}}$ and $\sigma_{F_{i}}$ be as in Theorem 3.1. Then $G$ is a permutation voltage lift of a bouquet $B$ of $k_{1}$ semiedges, labeled $1, \ldots, k_{1}$, and $k_{2}$ oriented loops, labeled $k_{1}+1, \ldots, k_{1}+k_{2}$, by the permutation voltage assignment in the group $\Gamma_{\mathcal{F}}$ given by $i \mapsto \delta_{F_{i}}$ for $i \in\left\{1, \ldots, k_{1}\right\}$ and $i \mapsto \sigma_{F_{i}}$ for $i \in\left\{k_{1}+1, \ldots, k_{2}\right\}$.

Note that Theorem 6 does not require any symmetry assumptions on the graph $G$. The permutation group $\Gamma_{\mathcal{F}}$ is known as the monodromy group of the associated covering of $B$ by $G$.

One may work in the reverse direction: Start with a bouquet $B$ of $k_{1}$ semiedges and $k_{2}$ loops, where $k=k_{1}+2 k_{2}$, and let $\Phi$ be a permutation voltage assignment on $B$ in a finite transitive permutation group $\Gamma$ that assigns involutions to semiedges. Unless the voltage assignment is chosen carefully, the (connected and $k$-regular) lift $B^{\Phi}$ is likely to have no nontrivial automorphisms. Even if some automorphisms exist, they may not arise "naturally" as automorphisms of the covering $\pi: B^{\Phi} \rightarrow B$ and are therefore hard to predict. Recall that $\alpha \in A u t\left(B^{\Phi}\right)$ is an automorphism of the covering $\pi$ if $\pi \alpha=\pi$, and the group of all such automorphisms is isomorphic to $N\left(\operatorname{Stab}_{\Gamma}(v)\right) / \operatorname{Stab}_{\Gamma}(v)$ for an arbitrary point $v$ of $B^{\Phi}[10]$.

Using the language of permutation voltage graphs, we also observe that the graph $G_{\Gamma}$ from Theorem 4 is a permutation voltage lift of a bouquet of cycles and semiedges under a permutation voltage assignment mapping the cycles and semiedges of the bouquet onto the permutations $\sigma_{x}, x \in X$, with the semiedges mapped to the involutions.

Next, we consider a specialization of the concept of a permutation voltage assignment lift to regular covers-covers distinguished by the existence of an automorphism group of the lifted graph that acts regularly on each fiber. As before, let $G=(V, E)$ be a finite graph with possible loops and multiple edges and $D(G)$ be the dart set of $G$. Given any (abstract) group $\Gamma$, we again define the voltage assignment $\Psi: D(G) \rightarrow \Gamma$ to be a mapping satisfying the property $\Psi\left(e^{-1}\right)=(\Psi(e))^{-1}$ for each $e \in D(G)$ (where $e^{-1}$ is the opposing dart to $e$ ). The voltage lift $G^{\Psi}$ is the graph with vertex set $V \times \Gamma$ with the adjacency relation connecting two vertices $\left(v_{1}, \gamma_{1}\right)$ and $\left(v_{2}, \gamma_{2}\right)$ if and only if there is a dart $e$ from $v_{1}$ to $v_{2}$ in $D(G)$ and $\gamma_{2}=\gamma_{1} \cdot \Psi(e)$.

In the case where one considers the left multiplication permutation action of a group $\Gamma$ on itself, the two concepts of a permutation voltage lift and the voltage lift coincide, but in general the class of permutation voltage lifts properly contains the class of voltage graph lifts. The relevance of voltage graphs to our construction is described in the following theorem. We leave the proof of this theorem to the reader again.

Theorem 7 Let $G$ be a $k$-regular graph whose edge set can be partitioned into a family $\mathcal{F}$ of 1 - and 2-factors. The Cayley graph $C a y\left(\Gamma_{\mathcal{F}}, Y\right)$ constructed in Theorem 3 is a connected component of the voltage graph lift $G^{\Psi}$ where $\Psi: D(G) \rightarrow \Gamma_{\mathcal{F}}$ maps a dart e to the generator $\sigma_{F_{i}}$ corresponding to the factor $F_{i}$ containing $e$. 
To summarize the above, we note that all our constructions are obtained from a bouquet of cycles and semiedges by first taking a permutation voltage lift and then taking a connected component of a voltage lift of the graph obtained in the first step.

\section{Smaller degree $k$ and girth $g$ Cayley graphs}

As the title of our paper suggests, we are ultimately interested in constructing $(k, g)$ Cayley graphs of the smallest possible orders.

Although the construction of Cayley graphs for any given degree $k$ and girth $g$ introduced in Sect. 2 is indeed explicit, the orders of the resulting graphs are rather hard to establish. The graphs we construct are Cayley graphs of permutation groups acting on sets of vertices of graphs that are truncations of $\left(k^{\prime}, g^{\prime}\right)$-graphs, where $k^{\prime}$ is equal to the order of a Cayley graph of degree $k-1$ and girth $g$, and $g^{\prime}$ is at least twice as big as $g$. As the size of these permutation groups may ultimately be approaching the factorial of the order of the truncated $\left(k^{\prime}, g^{\prime}\right)$-graphs, the sizes of Cayley graphs obtained in this way may be doubly exponential in the size of the $\left(k^{\prime}, g^{\prime}\right)$-graphs used to start the construction.

It is therefore meaningful to address the issue of constructing smaller Cayley graphs of given girth and degree than those constructed in Sect. 2.

As the order of any $(k, g)$-Cayley graph must certainly be at least as big as the order $n(k, g)$ of a $(k, g)$-cage, we recall here the well-known Moore bound $M(k, g)$.

$$
n(k, g) \geq M(k, g)= \begin{cases}1+k+k(k-1)+\cdots+k(k-1)^{(g-3) / 2}, & g \text { odd, } \\ 2\left(1+(k-1)+\cdots+(k-1)^{(g-2) / 2}\right), & g \text { even. }\end{cases}
$$

A $(k, g)$-cage whose order is equal to $M(k, g)$ is called a Moore graph, but Moore graphs are extremely rare, and the vast majority of $(k, g)$-cages are of order bigger than the Moore bound. If we denote the order of the smallest $(k, g)$-Cayley graph, the $(k, g)$-Cayley cage, by $n_{\text {cay }}(k, g)$, trivially

$$
n(k, g) \leq n_{\text {cay }}(k, g)
$$

for all $k \geq 2, g \geq 3$. Although a significant proportion of the known cages and current record-holders consists of Cayley graphs, the exact relation between $n(k, g)$ and $n_{\text {cay }}(k, g)$ is not known. In [8], the authors were able to prove that at least in some special cases (the degree of the graphs being a prime power), the order of the smallest $(k, g)$-Cayley graphs must differ from the Moore bound by at least the degree $k$. On the other hand, as pointed out at the beginning of this section, the order of the graphs constructed in the proof of Theorem 2 is essentially a double exponential of the Moore bound.

In order to construct smaller Cayley graphs, we take advantage of a result of Biggs [2] further generalized in [5]. For any given $k \geq 3$ and $g \geq 3$, Biggs constructs a group $\Gamma_{k, g}$ of girth at least $g$ generated by a set $X_{k, g}$ of $k$ involutions acting on a set of vertices of size roughly equal to $M(k, g)$. Although the exact order of the groups $\Gamma_{k, g}$ is again hard to establish in general, their size cannot exceed the factorial $M(k, g)$ ! (experiments with Biggs' construction suggest that this bound is often achieved). This 
means that the order of the groups $\Gamma_{k, g}$ is significantly smaller than the order of the groups we constructed in Sect. 2, and so the following constructions using the groups $\Gamma_{k, g}$ result in considerably smaller Cayley graphs.

We were unable to make these constructions work for some of the small parameter classes left out of the next theorem.

Theorem 8 For every pair $k, g$ satisfying one of the following conditions, there exists a $(k, g)$-Cayley graph of order equal to the order of a Cayley graph $\operatorname{Cay}\left(\Gamma_{k^{\prime}, g^{\prime}}, X_{k^{\prime}, g^{\prime}}\right)$ for appropriately chosen $k^{\prime}$ and $g^{\prime}$.

(i) $5 \leq g<k$,

(ii) $5 \leq k \leq g$,

(iii) $k=4, g \geq 4$ even.

Proof To construct a Cayley graph of degree $k$ and girth exactly $g$ for each of the three cases of our theorem, we consider Cayley graphs $\operatorname{Cay}(\Gamma, X)$ with $\Gamma$ being one of the groups $\Gamma_{k^{\prime}, g^{\prime}}$ and $X$ being an extension of the corresponding $X_{k^{\prime}, g^{\prime}}$. This will result in graphs of the same order, but higher degree, with the elements added to $X_{k^{\prime}, g^{\prime}}$ chosen as to allow us to prove the girth of the resulting graph to be exactly $g$. As this is already a second construction of Cayley graphs for the above parameters, we leave some details of the verification to the reader.

First assume that $5 \leq g<k$ or, equivalently, $4 \leq g-1 \leq k-2$. Let $\operatorname{Cay}\left(\Gamma_{k-2, r}\right.$, $\left.X_{k-2, r}\right)$ be Biggs' graph of degree $k-2$ of girth at least $r$, with $r$ greater than $(g-1)^{2}$. Then $X_{k-2, r}=\left\{x_{1}, x_{2}, \ldots, x_{k-2}\right\}$ with all $x_{i}$ being involutions. Let

$$
Y=X_{k-2, r} \cup\left\{x_{1} \cdot x_{2} \cdot \ldots \cdot x_{g-1}, x_{g-1} \cdot \ldots \cdot x_{2} \cdot x_{1}\right\} \text {. }
$$

Since no nonreversing product of elements in $X_{k-2, r}$ that is shorter than $(g-1)^{2}$ is equal to $1_{\Gamma_{k-2, r}}$ and $g-1<(g-1)^{2}$, the products $x_{1} \cdot x_{2} \cdot \ldots \cdot x_{g-1}$ and $x_{g-1} \cdot \ldots$. $x_{2} \cdot x_{1}$ are not equal to the identity. In addition, they are mutual inverses, and neither one is equal to any element in $X_{k-2, r}$. Thus, $Y$ does not contain the identity, is closed under inverses, and is of size $k$. Consequently, $G=\operatorname{Cay}\left(\Gamma_{k-2, r}, Y\right)$ is a Cayley graph of degree $k$. We claim that the girth of $G$ is exactly $g$. To see this, note first that the girth of $G$ is not bigger than $g$. This is due to the nonreversing identity

$$
x_{1} \cdot x_{2} \cdot \ldots \cdot x_{g-1} \cdot\left(x_{g-1} \cdot \ldots \cdot x_{2} \cdot x_{1}\right)=1_{\Gamma_{k-2, r}}
$$

that yields a cycle of length $g$ in $G$. To prove that the girth of $G$ is not smaller than $g$, let us suppose (by means of contradiction) the existence of a nonreversing identity

$$
y_{1} \cdot y_{2} \cdot \ldots \cdot y_{s}=1_{\Gamma_{k-2, r}},
$$

where $s<g$ and $y_{i} \in Y$ for all $1 \leq i \leq s$. Since the total number of symbols from $X_{k-2, r}$ involved in this identity is at most $s(g-1) \leq(g-1)^{2}$, the product $y_{1} \cdot y_{2}$. $\ldots y_{s}$ as a word in $X_{k-2, r}$ cannot be nonreversing, and thus, it must contain an element $x_{i} \in X_{k-2, r}$ followed by itself. Since $y_{1} \cdot y_{2} \cdot \ldots \cdot y_{s}$ is nonreversing as a word in $Y$, the repeated element must occur in one of the following four ways:

$$
y_{i}=x_{1} \cdot x_{2} \cdot \ldots \cdot x_{g-1}, y_{i+1}=x_{g-1} \text {, }
$$




$$
\begin{gathered}
y_{i}=x_{1}, y_{i+1}=x_{1} \cdot x_{2} \cdot \ldots \cdot x_{g-1}, \\
y_{i}=x_{g-1} \cdot \ldots \cdot x_{2} \cdot x_{1}, y_{i+1}=x_{1}, \\
y_{i}=x_{g-1}, y_{i+1}=x_{g-1} \cdot \ldots \cdot x_{2} \cdot x_{1},
\end{gathered}
$$

for some $1 \leq i<s$. After performing the cancellation of the two $x_{1}$ 's or the two $x_{g-1}$ 's, in order for the remaining word to be equal to $1_{G}$, we will need another pair of repeated elements from $X_{k-2, r}$ (involving a repeated $x_{2}$ or $x_{g-2}$ ). It is not hard to see now by induction that the minimum number of cancellations needed is at least $g-1$, and hence the identity $y_{1} \cdot y_{2} \cdot \ldots \cdot y_{s}$ must be of length at least $g$. This contradicts the choice of $s$ and completes the proof for the case $5 \leq g<k$.

Next assume that $5 \leq k \leq g$. Once again, let $\operatorname{Cay}\left(\Gamma_{k-2, r}, X_{k-2, r}\right)$ be Biggs' graph of degree $k-2$ and girth at least $r$ greater than $(g-1)^{2}$. As before, take $Y$ to be an extension of $X_{k-2, r}$ by a product of length $g-1$ and its inverse:

$$
\begin{aligned}
& x_{1} \cdot x_{2} \cdot \ldots \cdot x_{k-2} \cdot x_{1} \cdot x_{2} \cdot \ldots \\
& \ldots \cdot x_{2} \cdot x_{1} \cdot x_{k-2} \cdot \ldots \cdot x_{2} \cdot x_{1}
\end{aligned}
$$

(the product is obtained by repeatedly multiplying the elements of $X_{k-2, r}$ in the order $x_{1}, x_{2}, \ldots, x_{k-2}$ until reaching the desired length $g-1$; note that $k-2$ is assumed to be smaller than $g-1)$. The proof of the fact that $\operatorname{Cay}(G, Y)$ is a Cayley graph of degree $k$ and girth $g$ follows now along the same lines as the first half of the proof.

Finally, to show the existence of $(4, g)$-Cayley graphs, $g$ even, we use just a slight alteration of the previous proofs. Let $\operatorname{Cay}\left(\Gamma_{3, r}, X_{3, r}\right)$ be the 3-regular Cayley graph generated by three involutions $x_{1}, x_{2}, x_{3}$, that is, of girth $r>(g-1)^{2}$. For $g=4$, let

$$
Y=\left\{x_{1}, x_{2}, x_{3}, x_{1} \cdot x_{2} \cdot x_{1}\right\}
$$

for $g=6$, let

$$
Y=\left\{x_{1}, x_{2}, x_{3}, x_{1} \cdot x_{2} \cdot x_{3} \cdot x_{2} \cdot x_{1}\right\}
$$

for $g=8$, let

$$
Y=\left\{x_{1}, x_{2}, x_{3}, x_{1} \cdot x_{2} \cdot x_{3} \cdot x_{1} \cdot x_{3} \cdot x_{2} \cdot x_{1}\right\},
$$

for $g=10$, let

$$
Y=\left\{x_{1}, x_{2}, x_{3}, x_{1} \cdot x_{2} \cdot x_{3} \cdot x_{1} \cdot x_{2} \cdot x_{1} \cdot x_{3} \cdot x_{2} \cdot x_{1}\right\} .
$$

For $g \geq 12, Y$ can be obtained from $X_{3, r}$ by adding a palindrome $\left(x_{1} x_{2} x_{3}\right)^{m} \times$ $w\left(x_{3} x_{2} x_{1}\right)^{m}$ of length $g-1$, where $m=\left\lfloor\frac{g-2}{6}\right\rfloor$, and $w$ is one of the words $x_{1}, x_{1} x_{2} x_{1}$, $x_{1} x_{2} x_{3} x_{2} x_{1}$, dependently on the value of $\frac{g-2}{2}(\bmod 3)$. The added fourth element is in each case an involution of length $g-1$, and the proof of the 4-regular Cayley graphs $\operatorname{Cay}(G, Y)$ having girth $g$ follows along the same lines as above.

Notably, the above theorem does not cover the trivalent graphs. The majority of the missing trivalent graphs can be instead constructed by the truncation technique introduced at the end of the previous section. Namely, in order to construct a trivalent 
graph, one only needs to use 2-valent circulants of girth $g$, i.e., cycles of length $g$. Thus, the only ingredient needed to start the construction is the existence of a regular map of degree $g$ and girth at least $g / 2$. Nevertheless, the resulting graphs do not appear to be smaller than those constructed in Theorem 2.

\section{Small Cayley cages}

As stated at the beginning of the previous section, Moore graphs are rather rare. The sets of parameters $(k, 3),(k, 4), k \geq 3 ;(2, g), g \geq 3$; and $\left(p^{k}+1, g\right), p$ a prime and $g \in\{6,8,12\}$; are known to admit the existence of Moore graphs with the corresponding cages being the complete graph $K_{k+1}$, the complete bipartite graph $K_{k, k}$, the $g$-cycle $\mathcal{C}_{g}$, and the incidence graphs of projective planes, generalized quadrangles and generalized hexagons, respectively [5]. Almost all of the above graphs are also Cayley graphs:

(i) $K_{k+1} \cong \operatorname{Cay}\left(\mathbb{Z}_{k+1}, \mathbb{Z}_{k+1}-\{0\}\right)$ (or, for that matter, it is isomorphic to the Cayley graph of any group $G$ of order $k+1$, with the generating set consisting of all $k$ nonidentity elements of $G$ ),

(ii) $K_{k, k} \cong \operatorname{Cay}\left(\mathbb{D}_{2 k}, X\right)$ with $X$ consisting of the $k$ reflections of the dihedral group $\mathbb{D}_{2 k}$,

(iii) $\mathcal{C}_{g} \cong \operatorname{Cay}\left(\mathbb{Z}_{g},\{1, g-1\}\right)$,

(iv) vertex-transitive incidence graphs of projective planes have been shown to be Cayley graphs of dihedral groups in [9].

The general problem of the order of the smallest Cayley graph of given degree and girth appears at this point at least as hard as the general problem of the order of cages. In the concluding part of our paper, we list some few results concerning smallest $(k, g)$-Cayley graphs. All the constructive upper bounds in Table 1 have been shown to be best possible via exhaustive computer assisted search of all Cayley graphs with relevant parameters.

We bring the attention of the reader to the fact that the order of the smallest Cayley graphs for given degree and girth is (unlike the case of general cages) not monotonic with respect to the increase in $g$. Namely, the smallest $(3,6)-,(3,7)-$, and $(3,8)$ Cayley graphs are all smaller than the smallest $(3,5)$-Cayley graph. The situation repeats when the smallest $(3,12)$-Cayley graph is smaller than the smallest $(3,11)$ Cayley graph. Neither is the order monotonic in the degree: the order of the smallest $(3,5)$-Cayley graph is larger than the order of the smallest $(4,5)$-Cayley graph. However, deletion of two involutions or a pair of mutual inverses shows that

$$
n_{\text {cay }}(k, g) \leq n_{\text {cay }}(k+2, g)
$$

for all $k \geq 2, g \geq 3$. 
Table 1 List of small parameter pairs for which the Cayley cages are known

\begin{tabular}{|c|c|c|c|c|c|c|c|c|c|}
\hline \multirow[t]{2}{*}{ Girth } & \multicolumn{3}{|c|}{ Degree 3} & \multicolumn{3}{|c|}{ Degree 4} & \multicolumn{3}{|c|}{ Degree 5} \\
\hline & $\begin{array}{l}\text { Lower } \\
\text { bound }\end{array}$ & $\begin{array}{l}\text { Best } \\
\text { graph }\end{array}$ & $\begin{array}{l}\text { Cayley } \\
\text { cage }\end{array}$ & $\begin{array}{l}\text { Lower } \\
\text { bound }\end{array}$ & $\begin{array}{l}\text { Best } \\
\text { graph }\end{array}$ & $\begin{array}{l}\text { Cayley } \\
\text { cage }\end{array}$ & $\begin{array}{l}\text { Lower } \\
\text { bound }\end{array}$ & $\begin{array}{l}\text { Best } \\
\text { graph }\end{array}$ & $\begin{array}{l}\text { Cayley } \\
\text { cage }\end{array}$ \\
\hline 5 & 10 & 10 & 50 & 19 & 19 & 24 & 30 & 30 & 32 \\
\hline 6 & 14 & 14 & 14 & 26 & 26 & 26 & 42 & 42 & 42 \\
\hline 7 & 24 & 24 & 30 & 67 & 67 & 72 & 108 & 152 & 160 \\
\hline 8 & 30 & 30 & 42 & 80 & 80 & 96 & 170 & 170 & 288 \\
\hline 9 & 58 & 58 & 60 & 162 & 275 & 320 & 427 & & \\
\hline 10 & 70 & 70 & 96 & 243 & 384 & 410 & 683 & 1296 & \\
\hline 11 & 112 & 112 & 192 & 486 & & & 1707 & 2688 & \\
\hline 12 & 126 & 126 & 162 & 728 & 728 & & 2730 & 2730 & \\
\hline 13 & 202 & 272 & 272 & & & & & & \\
\hline 14 & 258 & 384 & 406 & & & & & & \\
\hline
\end{tabular}

Acknowledgements R. Jajcay supported by the projects APVV-0223-10 and VEGA 1/1005/12. J. Šrán̆ supported by the projects APVV-0104-07, APVV-0223-10, VEGA-1/0781/11 and APVV grant ESF-EC0009-10 within the EUROCORES Programme EUROGIGA (project GReGAS).

\section{References}

1. Biggs, N.L.: Girth and residual finiteness. Combinatorica 8, 307-312 (1988)

2. Biggs, N.L.: Constructions for cubic graphs of large girth. Electron. J. Comb. 5, A1 (1998)

3. Boben, M., Jajcay, R., Pisanski, T.: Generalized cages. Submitted for publication

4. Erdős, P., Sachs, H.: Reguläre Graphen gegebener Taillenweite mit minimaler Knotenzahl. Wiss. Z. Uni. Halle (Math. Nat.) 12, 251-257 (1963)

5. Exoo, G., Jajcay, R.: Dynamic cage survey. Electron. J. Comb. 15, DS16 (2008)

6. Gross, J.L.: Every connected regular graph of even degree is a Schreier coset graph. J. Comb. Theory, Ser. B 22(3), 227-232 (1977)

7. Gross, J.L., Tucker, T.W.: Topological Graph Theory. Wiley, New York (1987)

8. Jajcay, R., Širán̆, J.: Small vertex-transitive graphs of given degree and girth. Submitted for publication

9. Loz, E., Mačaj, M., Miller, M., Šiagiová, J., Širáň, J., Tomanová, J.: Small vertex-transitive and Cayley graphs of girth six and given degree: an algebraic approach. To appear

10. Massey, W.S.: Algebraic Topology: An Introduction. Graduate Texts in Mathematics, vol. 56. Springer, New York (1981)

11. Nedela, R., Škoviera, M.: Which generalized Petersen graphs are Cayley graphs? J. Graph Theory 19(1), 1-11 (1995)

12. Sachs, H.: Regular graphs with given girth and restricted circuits. J. Lond. Math. Soc. 38, 423-429 (1963)

13. Šiagiová, J., Watkins, M.E.: Covalence sequences of planar vertex-homogeneous maps. Discrete Math. 307(3-5), 599-614 (2007)

14. Širáň, J., Škoviera, M.: Quotients of connected regular graphs of even degree. J. Comb. Theory, Ser. B 38, 214-225 (1985) 